\title{
Challenges
}

\author{
Marvin L. Birnbaum, MD, PhD
}

A principal function of an International Congress attended by a host of the world's experts in Disaster Medicine is to provide a forum for the exchange of experiences relative to the occurrence of and responses to catastrophes world-wide. Based upon the abstracts submitted to the 7th Congress, there is a profound paucity of such descriptions. The absence of such materials leaves me in a quandary whether I should be pleased or disturbed. Perhaps, it means that there only have been a few disasters since the 6th Congress and that the United Nations (UN) has achieved its goal of making the 1990s the "Decade for the Reduction of Natural Disasters." But, there have been severe earthquakes in the People's Republic of China, typhoons in Bangladesh, floods in Thailand, drought and famine in the Sudan, war in the Middle East and Africa that have produced huge refugee populations, hurricanes and tornados in North America, and industrial accidents world-wide. I suspect that the need for the exchange of information and the responses to them has been neglected out of a failure to develop the standard language required to describe such events in a meaningful way. Perhaps, it results from so many uncoordinated organizations, each perceiving its principal mission to prevent or respond to such events while protecting its respective autonomy from intrusion. Perhaps, this is an expression of the failure of international organizations to provide the leadership necessary to establish priorities and to stimulate optimal use of limited resources. Priorities for the use of such resources remain undefined.

Definitions: Several authors in this issue have indicated that Disaster Medicine will not become a science until we establish and agree on our own language. So far, no such standard and accepted definitions exist. For example, several definitions of "disaster" (catastrophe) have been suggested within this very issue. The world needs consensus on definitions for: disaster; its types, scope, phases; triage classifications for use in the field, during conveyance, and in the receiving facilities; injury descriptors; a severity scoring system; and, the elements of a minimum data base. Therefore, I challenge the delegates at the 7th World Congress to develop and approve such a set of universal definitions before they leave Montreal.

Parochialism: The failure to share information because of the parochial needs of the numerous organizations involved, is not difficult to understand, but it has created great difficulty in the sharing of experiences. There is so much we all know so little about. Hopefully, the Federation of Societies for Emergency and Disaster Medicine will help to meet this need for communications without any sacrifice of the autonomy of its component societies. The Federation must encourage translation of information into other languages so that we can share our specific bits of information and strengthen our knowledge. I challenge the new Federation to organize these efforts, to promote the distribution of this information, and to use Prehospital and Disaster Medicine to serve as the organ for these activities.

International Agenda: The collective talents, energy, and wisdom of the members of the World Association for Emergency and Disaster Medicine (WAEDM) and other organizations represented at the 7th Congress are the best in the World. But, we must know where to place our energies in order to optimize our impact. We need to offer our assistance to the United Nations in the development and implementation of programs which will mitigate the occurrence and the effects of Natural Disasters. We comprise the medical expertise needed to accomplish this mission. We are in this together and without us, this noble objective will not be achievable. I challenge WAEDM and the U.N. to come together to establish a common agenda. Thereto, I offer the medium of this Journal to assist the United Nations in the distribution of the agenda for this important mission.

These issues are clear and the stage is set. I look forward to the performance and gratefully will lead the applause. 
Identification of $\mathrm{CO}_{2}$ immediately following intubation is fast and simple with the MiniCAP ${ }^{\mathrm{M}} \mathrm{III} \mathrm{CO}_{2}$ detector.

\section{Take the guesswork out of intubation.}

Using sophisticated infrared technology connected directly to the endotracheal tube, the MiniCAP III detects the presence and absence of $\mathrm{CO}_{2}$ during the respiration cycle. A loss of respiration alarm is provided, adjustable from 5-60 seconds.

Unlike disposable chemical-type detectors, the MiniCAP III uses bright LEDs and an audible tone to indicate a complete respiratory cycle under any ambient light condition. The MiniCAP III also provides visual and audible alarms for loss of respiration, which chemical-type detectors can't provide.

Rugged and reliable, the battery-powered MiniCAP III CO $\mathrm{CO}_{2}$ detector belongs with every intubation kit on crash carts or in ambulances. Don't settle for anything less.

For further details on the $\mathrm{CO}_{2}$ detector you can use with confidence, call 1-800-672. 4678, Ext. 8826

\section{MCA CATALYST RESEARCH}

3706 Crondall Lane

Owings Mills. MD 21117 $301 / 356-2400$

\section{MSA GATALYST}

- LOW

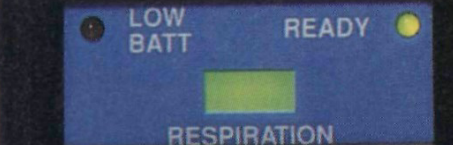

